\title{
Converging low energy expansion of nucleon-nucleon scattering based on the Wilsonian renormalization group analysis
}

\author{
Hirofumi Kubo*i \\ E-mail: kubodhiggs.phys.kvushu-u.ac.jp
}

Koji Harada

E-mail: haradadphys.kyushu-u.ac.jp

Tatsuya Sakaeda ${ }^{\S}$

E-mail: sakaedadhigqs.phys.kyushu-u.ac.jp

Yuki Yamamoto"I

E-mail: vamamotodkoeki-u.ac.jp

In order to implement the power counting obtained in the Wilsonian renormalization group (WRG) analysis of nuclear effective field theory (NEFT) including pions, we propose a practical calculational scheme in which the short-distance part of one-pion exchange (S-OPE) is removed and represented as contact terms. A distinctive feature of our scheme is that two regularization schemes are used in combination. The dimensional regularization (DR) is used for diagrams consisting only of contact interactions and a momentum cutoff is implemented for diagrams containing the long-distance part of one pion exchange (L-OPE) by introducing a Gaussian damping factor(GDF). The sole purpose of the use of the dimensional regularization is that it considerably simplifies the treatment of nonperturbative part of the scattering amplitude. In the calculation the L-OPE is treated as perturbation and a part of the S-OPE is treated nonperturbatively along with a contact interaction without derivative nonperturbatively. We show the results of the next-to-nextto-leading order(NNLO) calculations for nucleon-nucleon elastic scattering in the S-waves that are fitted to Nijmegen partial wave analysis data.

XV International Conference on Hadron Spectroscopy-Hadron 2013

4-8 November 2013

Nara, Japan

\footnotetext{
* Speaker.

${ }^{\dagger}$ Synchrotron Light Application Center, Saga University 1 Honjo, Saga 840-8502, Japan

${ }^{\ddagger}$ Faculty of Arts and Science and Department of Physics, Kyushu University Fukuoka 810-8581 Japan

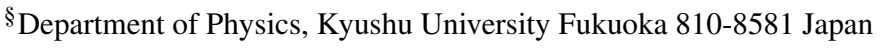

IDepartment of Community Service and Science, Tohoku University of Community Services and Science Iimoriyama 3-5-1, Sakata 998-8580 Japan
} 


\section{Introduction}

Nuclear effective field theory(NEFT) is one of the most promising approaches to the modelindependent description of interactions between nucleons at low energies. There has been a tremendous amount of applications with great success. See Ref. [四 for a recent review. However, there are still remaining issues related to the power counting, which is necessary to organize the infinitely many contributions arising from the most general Lagrangian.

In order to calculate physical quantities one needs to establish a power counting scheme. Two distinct power counting schemes are proposed for nuclear EFT: Weinberg power counting scheme and Kaplan, Savage, and Wise(KSW) power counting. The most distinctive difference between them is the treatment of pion exchanges. In Weinberg power counting pion exchanges are treated nonperturbatively along with all the contact terms. On the other hand in KSW power counting pion exchanges are treated as perturbations.

The Weinberg power counting is widely used in most of numerical calculations with successful agreement with experimental data, but it is shown to be inconsistent, i.e., cutoff dependence arising at a certain order can be absorbed only by terms of higher orders [[]]. Several authors (e.g. [3]) consider variants of the Weinberg's scheme and discuss the nonperturbative renormalization of them.

On the other hand the KSW power counting is shown to be consistent in regards to renormalization. However, it has been shown by Fleming and his collaborators that the low energy expansion of nucleon-nucleon scattering amplitude in the spin-triplet channel employing KSW power counting breaks down at next-to-next-to-leading order[团]. After that almost all the NEFT calculations are done employing Weinberg power counting, which treats pion exchanges nonperturbatively.

\section{Wilsonian RG and power counting}

In Ref.[[] we proposed a way to determine the power counting systematically through Wilsonian renormalization group (WRG) analysis, which is a nonperturbative method. The basic idea of the power counting is the order of magnitude estimate based on dimensional analysis. The scaling dimension is the quantum counterpart of the canonical dimension, which is determined by WRG analysis. A nonperturbative RG analysis is necessary because in the $\mathrm{S}$ waves the physical two-nucleon system is governed by a nontrivial fixed point which is inaccessible in perturbation theory.

In Ref.[回], we applied the Wilsonian RG idea to the NEFT including pions. In order to understand the implication of the results it is important to remember that pion exchange is divided into its short-distance part (S-OPE) and the long-distance part (L-OPE) separated by a cutoff scale. The S-OPE is represented as contact terms. A part of the S-OPE is relevant in the RG sense and thus should be treated nonperturbatively while the L-OPE is treated as perturbation.

\section{A hybrid regularization}

In implementing the power counting suggested by the WRG analysis correctly, we stress that it is necessary to separate the pion exchange into the short-distance part (S-OPE) and the long- 
Table 1: Dimensionless coupling constants fitted to the weighted Nijmegen PWA data in the ${ }^{1} S_{0}$ channel.

\begin{tabular}{c|llllll} 
& $\tilde{C}_{0}^{\left(1 S_{0}\right)}$ & $\tilde{C}_{2}^{\left(1 S_{0}\right)}$ & $\tilde{D}_{2}^{\left({ }^{1} S_{0}\right)}$ & $\left.\tilde{C}_{4}^{(1} S_{0}\right)$ & $\tilde{D}_{4}^{\left({ }^{1} S_{0}\right)}$ & $\tilde{E}_{4}^{\left({ }^{1} S_{0}\right)}$ \\
\hline LO & -0.958 & - & - & - & - & - \\
NLO & -0.964 & 0.517 & 0.479 & - & - & - \\
NNLO & -0.967 & 0.205 & 0.099 & -0.055 & 1.180 & 1.203
\end{tabular}

Table 2: Dimensionless coupling constants fitted to the weighted Nijmegen PWA data in the ${ }^{3} S_{1}{ }^{3} D_{1}$ channel.

\begin{tabular}{c|lllllll} 
& $\left.\tilde{C}_{0}^{(3} S_{1}\right)$ & $\left.\tilde{C}_{2}^{(3} S_{1}\right)$ & $\left.\tilde{D}_{2}^{(3} S_{1}\right)$ & $\left.\tilde{C}_{4}^{(3} S_{1}\right)$ & $\left.\tilde{D}_{4}^{(3} S_{1}\right)$ & $\left.\tilde{E}_{4}^{(3} S_{1}\right)$ & $\tilde{C}_{2}^{(S D)}$ \\
\hline LO & -1.22 & - & - & - & - & - & - \\
NLO & -1.31 & 1.47 & 0.68 & - & - & - & - \\
NNLO & -1.42 & 0.89 & -0.11 & -11.72 & 4.28 & 31.1 & -4.80
\end{tabular}

distance part (L-OPE) because the former contains contributions that need to be treated nonperturbatively while the latter should be treated perturbatively.

In order to put this idea into an actual calculation, we propose a practical calculational scheme using a hybrid regularization in which diagrams consisting only of contact interactions are regularized with dimensional regularization(DR) with power divergence subtraction, in which a scale $\mu$ is introduced and those including pion exchanges are regularized introducing a Gaussian damping factor(GDF) in which a scale $\lambda$ is introduced.

Many applications of EFT including Ref.[䧃] calculations are performed using DR, which is a very convenient regularization scheme for doing loop calculations analytically. One distinguishing feature is that the operator mixing is minimal. As a result of using DR the relevant operator in NEFT is represented by the four-nucleon operator without derivative. This is in sharp contrast to the momentum cutoff regularization. One might be convinced that it is a sufficient scheme for loop calculations in NEFT including pions. Although the problem of infinities do not appear we suspect that this method does not properly separate the short-distance singularity such as $1 / r^{3}$ for $r \rightarrow 0$, which appears in spin-triplet channels due to tensor interactions. We suspect that this is the reason for the nonconvergence problem reported in Ref.[䧃].

\section{Results and conclusion}

We calculated the LO, NLO, and NNLO amplitudes for the nucleon-nucleon scattering in the ${ }^{1} S_{0}$ and ${ }^{3} S_{1}-{ }^{3} D_{1}$ channels analytically using the hybrid regularization and fit the phase shifts obtained from the amplitudes to the Nijmegen PWA data. In our calculation we include diagrams containing an $S-D$ mixing contact term together with the diagrams given in Ref. [四].

In our approach, the "renormalization scale" $\mu$ appearing in the PDS is identified with the scale $\lambda$ up to a numerical constant. The scale $\lambda$ plays an analogous role to that of the floating cutoff in the Wilsonian RG analysis. The low-momentum physical quantities should not depend on the values of $\lambda$. This requirement leads to a set of renormalization group equations (RGEs) for the coupling constants. The requirement is satisfied order by order. We confirmed that our analytic expressions for the scattering amplitudes are independent of scales $\mu$ and $\lambda$ provided the coupling 


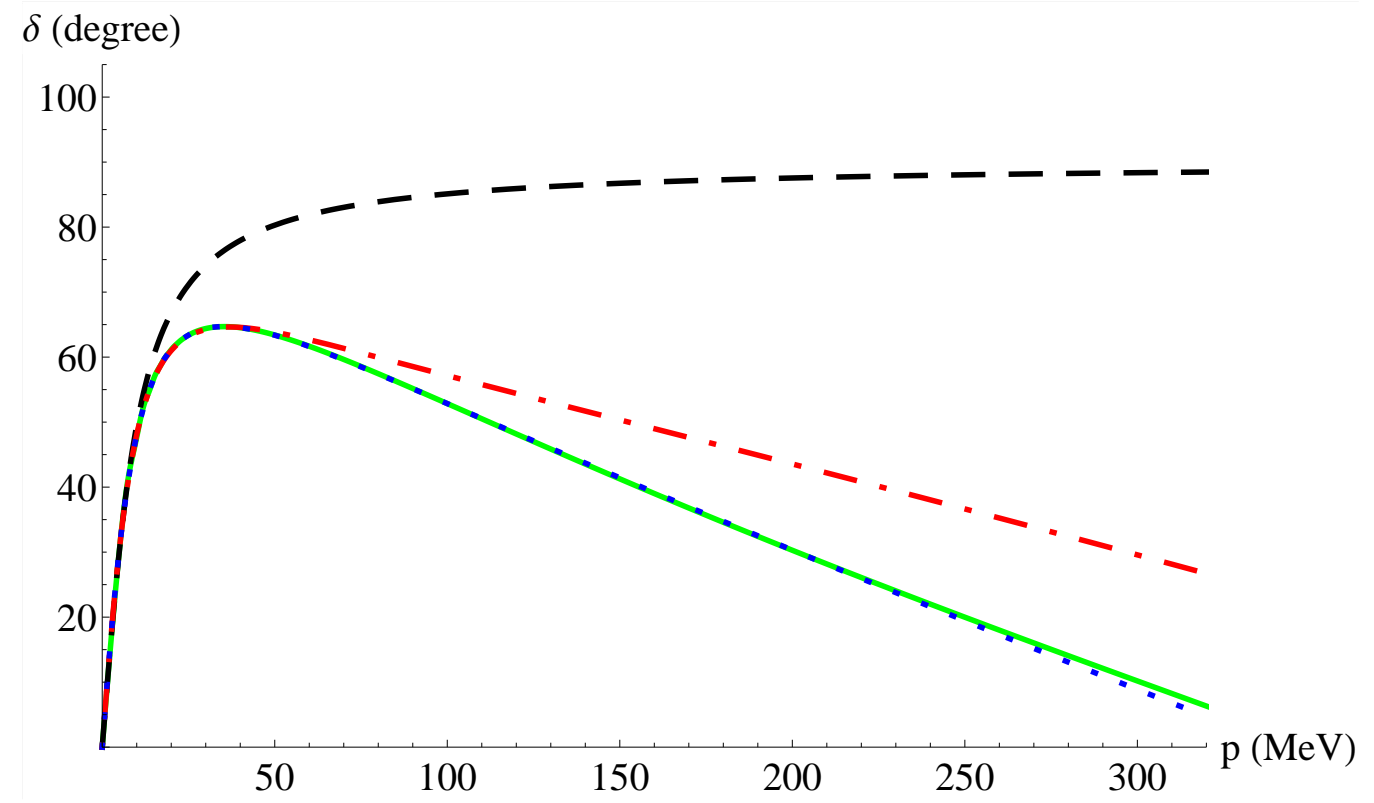

Figure 1: The scattering phase shift for the ${ }^{1} S_{0}$ channel. The LO(dashed line), NLO(dash-dotted line), and NNLO(dotted line) results are plotted together with the Nijmegen PWA data(solid line).

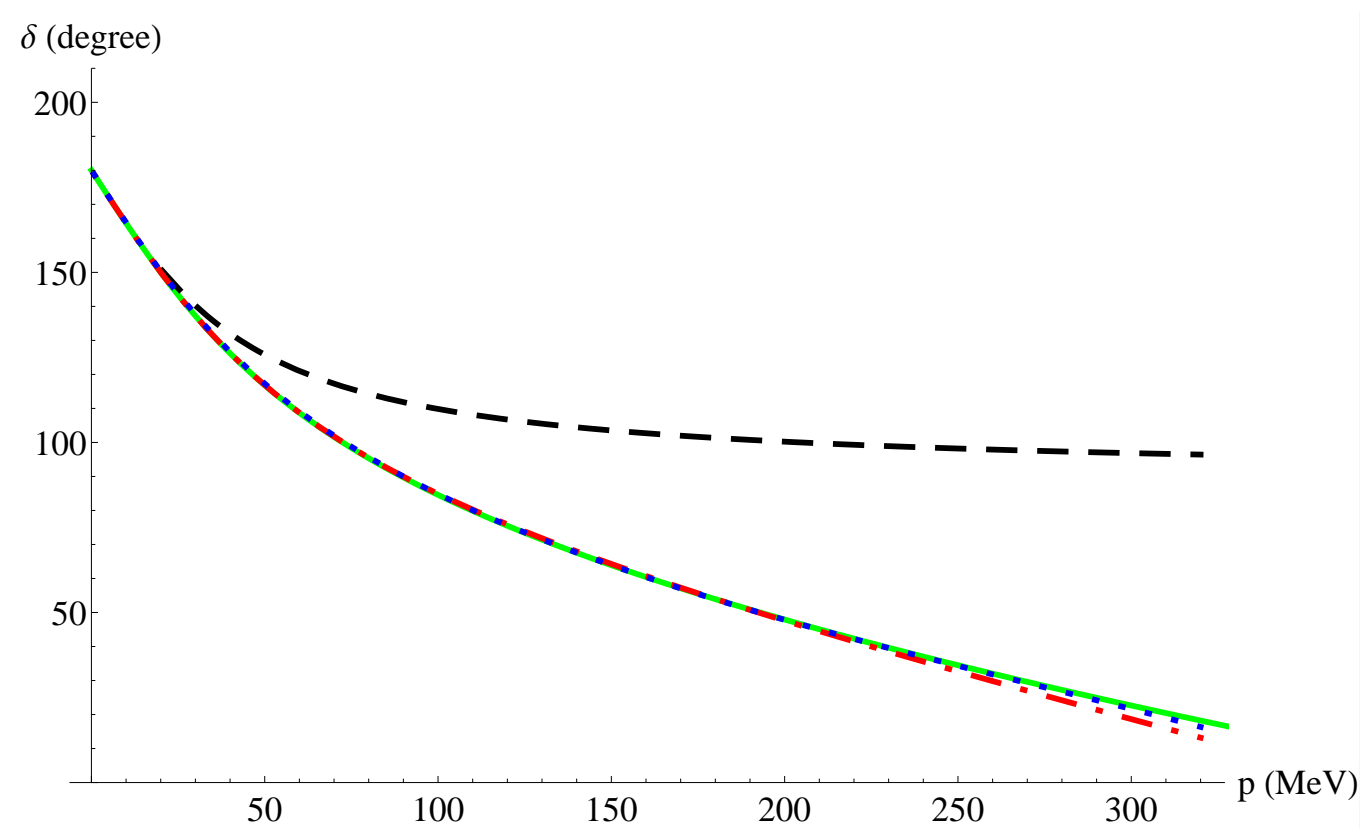

Figure 2: The scattering phase shift for the ${ }^{3} S_{1}$ channel.The LO(dashed line), NLO(dash-dotted line), and NNLO(dotted line) results are plotted together with the Nijmegen PWA data(solid line). 
constants satisfy the RGEs. In deriving the RGEs we also found that a careful redefinition of the pion exchange coupling constant is necessary in order for the amplitude to be independent of the cutoff. This finding is possible only with analytic expressions and the RG analysis. We think that it is also important for numerical studies with similar damping factors.

We only show the fitted results here. The results for ${ }^{1} S_{0}$ channel and ${ }^{3} S_{1}$ channel are shown

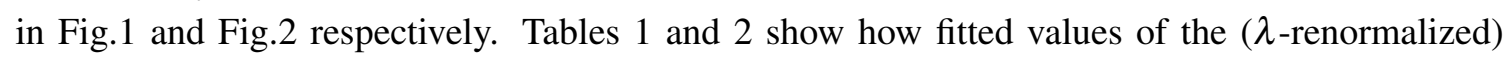
dimensionless coupling constants change as we go higher orders.

The fitted values of most of the coupling constants are of the natural size and the effective field theory expansion seems converging up to including the NNLO. Some of the details of our calculation are explained in Ref. [四].

\section{References}

[1] Evgeny Epelbaum, Hans-Werner Hammer, and Ulf-G. Meissner, Modern Theory of Nuclear Forces, Rev.Mod.Phys. 81:1773-1825, 2009.

[2] David B. Kaplan, Martin J. Savage, and Mark B. Wise, Nucleon-Nucleon Scattering from Effective Field Theory. Nucl. Phys. B478:629-659, 1996.

[3] A. Nogga, R.G.E. Timmermans, and U. van Kolck, Renormalization of One-Pion Exchange and Power Counting., Phys.Rev. C72:054006, 2005.

[4] Sean Fleming, Thomas Mehen, and Iain W. Stewart, NNLO corrections to nucleon nucleon scattering and perturbative pions, Nucl. Phys. A677:313-366, 2000.

[5] Koji Harada, Hirofumi Kubo, Anomalous dimensions determine the power counting: Wilsonian RG analysis of nuclear EFT, Nucl.Phys B758:304-329, 2006.

[6] Koji Harada, Hirofumi Kubo and Yuki Yamamoto, Pions are neither perturbative nor nonperturbative: Wilsonian renormalization group analysis of nuclear effective field theory including pions, Phys.Rev. C83:034002, 2011.

[7] Koji Harada, Hirofumi Kubo, Tatsuya Sakaeda and Yuki Yamamoto, Convergent perturbative nuclear effective field theory, arXiv:1311.3063. 